\title{
Evolution of abundance gradients for galactic plane $\mathrm{PNe}$
}

\author{
A.F. Kholtygin ${ }^{1}$, Yu.V. Milanova \\ and V.V. Akimkin \\ Department of Astronomy, Saint-Petersburg State University, Saint-Petersburg, Russia \\ ${ }^{1}$ email: afkholtygin@gmail.com
}

\begin{abstract}
The modern observations of planetary nebulae (PNe) are used to recalculate the element abundances for more than 150 PNe of the Milky Way and Magellanic Clouds. Basing on our data, we study the evolution of the abundance gradients for PNe in the thin disk and in the bulge.
\end{abstract}

A homogeneous set of the nebular parameters and element abundances for more than 300 galactic PNe (Milanova \& Kholtygin 2009) was compiled using our calculations and literature data. These data were used to create a new catalogue of planetary nebulae (www.astro.spbu.ru/staff/afk/GalChemEvol) which contains the parameters of Galactic and Magellanic Cloud PNe. To study the evolution of the abundances in the Galactic disk we normalized the different distances found in literature catalogues using the well known distance to the Galactic center (Nikiforov 2004). The comparison of our data (Akimkin et al. 2009, in prep.) with those given by Stanghellini et al. 2008 is shown in Fig. 1.

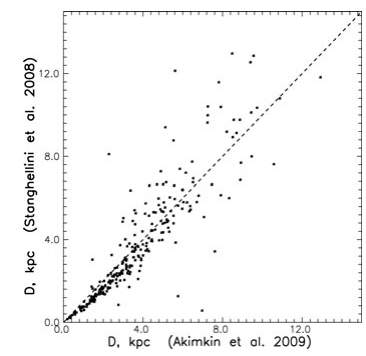

Figure 1. Our distances compared with those from Stanghellini et al. (2008)

We have found the regular increasing of the $\mathrm{Ne}$ and $\mathrm{Cl}$ abundance on the disk PNe with the age of the progenitor stars, but did not find clear evidence for the evolutionary changes of the radial abundances gradients for $\mathrm{O}$ and $\mathrm{S}$. We compared the abundances for the different galactic subsystems and detected that abundance patterns are similar for bulge PNe and Peimbert's type II nebulae, for which progenitor stars belong to the population of the thin disk with the ages greater than 4-6 Gyrs. We suppose that the intense star formation both in the bulge and in the thin disk continued at least up to 4-6 Gyr ago.

\section{References}

Milanova, Yu. V. \& Kholtygin A. F. 2009, Astronomy Letters, 35, 518

Nikiforov, I. 2004, ASP Conf. Series, 316

Stanghellini, L. Shaw, R. A., \& Villaver, E. 2008, ApJ 689, 194 